\title{
Investigation of in vivo protective effect of orally administered vitamin $E$ and selenium against gentamicin- induced renal and hepatic toxicity
}

\author{
Amin A Al-Doaiss ${ }^{1,2}$, Yazun B Jarrar ${ }^{3 *}$ \\ ${ }^{1}$ Department of Biology, College of Science, King Khalid University, Abha, Saudi Arabia, ${ }^{2}$ Anatomy and Histology Department, \\ Faculty of Medicine, Sana'a University, Sana'a, Republic of Yemen, ${ }^{3}$ Department of Pharmacy, College of Pharmacy, \\ Alzaytoonah University of Jordan, Amman, Jordan
}

*For correspondence: Email: yazun.jarrar@zuj.edu.jo; Tel: 00962-795930283

Sent for review: 21 February 2019

Revised accepted: 21 June 2019

\begin{abstract}
Purpose: To investigate the protective effect of vitamin E (Vit E) and selenium (Se) combination against gentamycin (GM)-induced renal and hepatic toxicity in rats.

Methods: Forty-eight male Wistar albino rats were administrated GM at a dose of $80 \mathrm{mg} / \mathrm{kg} / \mathrm{day}$, with or without Se $(1.5 \mathrm{mg} / \mathrm{kg} /$ day $)$, and/or Vit E (250 mg/kg/day) for a period of 4 weeks. Serum samples from each rat were subjected to biochemical analysis for kidney and liver functions, while kidney and liver biopsies were also investigated by histological examination.

Results: GM significantly increased serum creatinine, urea, alanine aminotransferase (ALT), aspartate aminotransferase (AST) and free radicals $(p<0.05)$. Moreover, GM induced significant histological and ultrastructural alterations in the renal and hepatic tissues of the rats. Exposure to a combination of Vit $E$ and Se did not attenuate the GM-induced toxicity in renal and hepatic tissues.

Conclusion: These results suggest that Vit $E$ and Se combination have no significant protective role against GM-induced hepatic and renal toxicity.
\end{abstract}

Keywords: Antioxidants, Vitamin E, Selenium, Gentamicin, Toxicity

\begin{abstract}
This is an Open Access article that uses a fund-ing model which does not charge readers or their institutions for access and distributed under the terms of the Creative Commons Attribution License (http://creativecommons.org/licenses/by/4.0) and the Budapest Open Access Initiative (http://www.budapestopenaccessinitiative.org/read), which permit unrestricted use, distribution, and reproduction in any medium, provided the original work is properly credited.

Tropical Journal of Pharmaceutical Research is indexed by Science Citation Index (SciSearch), Scopus, International Pharmaceutical Abstract, Chemical Abstracts, Embase, Index Copernicus, EBSCO, African Index Medicus, JournalSeek, Journal Citation Reports/Science Edition, Directory of Open Access Journals (DOAJ), African Journal Online, Bioline International, Open-J-Gate and Pharmacy Abstracts
\end{abstract}

\section{INTRODUCTION}

Gentamicin (GM) is still considered an important aminoglycoside antibiotic which is widely used in treatment of bacterial infections caused by gram negative bacteria [1]. It is used clinically in treatment of urinary tract infections and endocarditis [2]. However, treatment with this antibiotic causes renal and hepatic toxicity through induction of oxidative stress, apoptosis and necrosis [3]. The GM-induced nephrotoxicity is related to its accumulation in the renal proximal convoluted tubules leading to acute renal failure and tubular necrosis [4].

Extracts of medicinal plants, trace elements, vitamins and antioxidants have been successfully used to ameliorate GM-induced toxicity [5-7]. Several approaches have been attempted for reducing GM toxicity. These efforts were mainly focused on the use of plant antioxidant extracts [6] or other agents with antioxidant properties [8]. 
Vitamin E (Vit E) and selenium (Se) are essential nutrients which function as antioxidants and hence minimize cellular damage caused by free radicals such as reactive oxygen species and endogenous peroxides [9]. Vitamin $\mathrm{E}$ and $\mathrm{Se}$ prevent oxidative damage to the cellular membrane by decreasing hydroperoxide formation [10]. Previous studies have reported the nephro-protective effects of Vit $E$ and $S e$ in rats fed high-cholesterol diets [11], and in organo-phosphate-induced toxicity [12] and cadmium-induced renal toxicity [13]. In addition, Vit $\mathrm{E}$ and $\mathrm{Se}$ are protective against malathioninduced hepatotoxicity [14]. However, Vit E, in combination with diphenyl-phenylenediamine, did not exert protective effects against GM-induced nephrotoxicity [15]. Indeed, a study has reported that Vit $E$ increased the risk of cancer development [16].

Several studies have reported the nephroprotective and hepatoprotective effects of Vit $E$ and Se, but the effect of Vit $E$ and Se combination on GM-induced nephrotoxicity and hepatotoxicity has not been investigated. Therefore, the present study was conducted to determine the in vivo influence of orallyadministered Vit $\mathrm{E}$ and $\mathrm{Se}$ on GM-induced toxicity in renal and hepatic tissues.

\section{EXPERIMENTAL}

\section{Chemicals and drugs}

Gentamicin was obtained from Al-Qassim Pharmaceutical Company of Saudi Arabia. Vitamin E ( $\alpha$-tocopherol, Evion $100 \mathrm{mg}$ capsule) and Se were purchased from Merck Limited Company, Germany, and Oxoid Limited Company (Basingstoke, UK), respectively.

\section{Experimental animals}

Adult male Wistar albino rats weighing $220-250$ $\mathrm{g}$ were obtained from the Animal Care Centre in College of Pharmacy, King Saud University. All rats were housed under controlled conditions which included controlled suitable temperature $\left(22 \pm 1^{\circ} \mathrm{C}\right)$ and a $12 \mathrm{~h}$ light/12h dark cycle. The rats were fed laboratory animal diet and water ad libitum. The protocol for the current study was approved by the Research Ethics Committee of College of Pharmacy, King Saud University, with reference number 97-40. All the experimental procedures were conducted in the Histology and Cell Biology Laboratory at King Saud University, Saudi Arabia. The rats were handled and treated according to the guidelines of Canadian Council on Animal Care [17].

\section{Experimental design}

Forty-eight male Wistar albino rats (Rattus norvegicus) were randomly assigned to four groups of 12 rats each as follows:

Control: Each rat received daily $1 \mathrm{~mL}$ of $0.9 \%$ saline and $0.25 \mathrm{~mL}$ corn oil via gavage for a period of 4 weeks.

GM-treated: The rats were given GM at a dose of $80 \mathrm{mg} / \mathrm{kg} /$ day via intramuscular (im) injection for a period of 4 weeks.

GM-treated group with co-administration of Vit $\mathrm{E}$ and Se: Single daily doses of Se $(1.5 \mathrm{mg} / \mathrm{kg})$ and Vit $E(250 \mathrm{mg} / \mathrm{kg}$, dissolved in corn oil) were administrated to the rats via gavage. One hour later, GM was given at a dose of $80 \mathrm{mg} / \mathrm{kg}$ via im injection for 4 weeks.

Vitamin E- and Se-treated: The rats in this group received daily single dose of Se $(1.5 \mathrm{mg} / \mathrm{kg})$ and single dose of Vit $E(250 \mathrm{mg} / \mathrm{kg})$ via gavage for 4 weeks. The doses of GM, Vit E and Se were based on previous investigations $[7,12,14]$.

\section{Biochemical analyses}

At the end of the first and fourth weeks after treatment with GM and combination of Vit $E$ and Se, blood samples were taken from the orbital sinus of each rat, and serum was obtained for measurement of biochemical parameters of kidney function (urea and creatinine), and liver function (ALT, AST, and alkaline phosphatase, ALP). A blood Chemical Analyser (Reflotron, Roche Co., Germany) was used for the biochemical analysis, using the appropriate assay kits. The levels of free radicals in rat blood were determined with FRAS-4 (Iram-Param Co., Italy), as described previously [14].

\section{Histopathological examination}

Six rats from each group were sacrificed. The kidney and liver specimens were excised for histological examination at the end of first and fourth weeks of treatment with GM and the combination of Vit $\mathrm{E}$ and Se. Fresh biopsies of both kidney and the liver from each rat were taken and fixed in neutral buffered formalin (10 $\%$ ), followed by dehydration in ascending concentrations of ethanol (70 to $100 \%$ ). Dehydration was followed by clearing in xylene. Then, the tissue samples were impregnated in molten paraffin wax, embedded and blocked. Thereafter, 4- $\mu \mathrm{m}$ tissue sections were sliced with a microtome and stained with hematoxylin and 
eosin $(H$ \& $E)$. Histological changes were observed and photographed under an Olympus Microscope BX51 with Digital Camera (Japan).

\section{Ultrastructural analysis}

For ultrastructural analysis, small, fresh tissue blocks $(1-3 \mathrm{~mm} 3)$ were cut off from the kidney and liver tissues. Tissue blocks were fixed in $3 \%$ glutaraldehyde solution which was embedded in epoxy resin and processed for ultrastructural analysis using electron microscopy.

\section{Statistical analysis}

Data are presented as mean \pm standard deviations (SD). The data were statistically analysed with SAS, 2002, using one-way ANOVA test. Statistically significant changes in comparison with the control group were indicated as $p<0.05$.

\section{RESULTS}

\section{Effect of Vit $E$ and Se on GM-induced biochemical alterations}

Table 1 shows that GM significantly elevated serum ALT, ALP, AST, urea and creatinine levels after 1 and 4 weeks of exposure ( $p$ value $<0.05$ ). Co-administration of vitamin $E$ and Se did not ameliorate the GM-induced changes in kidney and liver functions tests. Indeed, after 4 weeks of treatment of the GM rats with Vit E and Se, ALT and AST were more significantly elevated, relative to rats treated with GM alone $(p<0.05)$. On the other hand, Vit E + Se- treated rats had significantly lower levels of free radicals $(p<$ 0.05 ) after 1 and 4 weeks of administration, when compared with the GM-treated rats and GM rats that received co-administration of Vit $\mathrm{E}$ and Se.

\section{Effect of Vit E and Se on GM-induced renal histological alterations}

The kidneys of control rats showed normal histological features of glomerular, tubular and interstitial components of the cortex and the medulla (Figure 1 a). In addition, ultra-thin sections of the control kidneys showed normal ultrastructure (Figure $1 \mathrm{~b}$ ). The epithelial lining of the PCTs had normal profuse apical microvilli and spherical nuclei with euchromasia. Several mitochondria were observed in the cytoplasm of the renal cells.
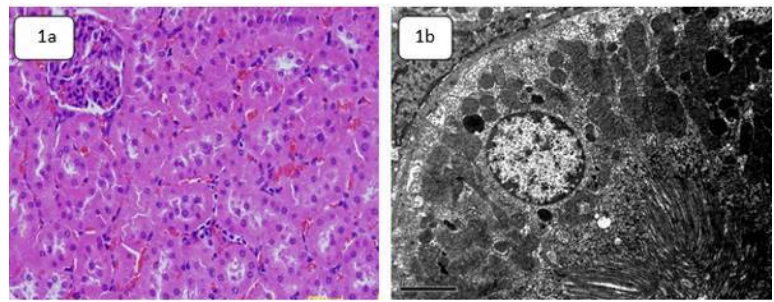

Figure 1: (a) Photomicrograph of kidney section of control rat demonstrating normal structures $(H$ \& $E$, $\times 400$ ); (b) Electron micrograph of kidney of control rat with normal ultrastructure. Note the spherical nucleus with euchromasia, together with normal mitochondria and profuse apical microvilli $(\times 5000)$.

There were no abnormalities in the histology and ultrastructure of the kidneys from rats treated with Vit. E and Se. In contrast, GM induced marked tubular and interstitial alterations in the kidneys of rats in the GM-treated group. The tubular alterations due to GM treatment appeared early in the form of necrosis, degeneration and vacuolization by the end of the first week. The degenerative tubules had swelling and loss of the proximal tubular brush border. These changes were seen in most of the proximal convoluted tubules, and to a lesser extent in the distal tubules (Figure 2a). Severe tubular necrosis occurred and intertubular lymphocytic infiltration appeared after one week of GM treatment at dose $80 \mathrm{mg} / \mathrm{kg}$ (Figures $2 \mathrm{~b}$ and $2 \mathrm{c}$ ). Moreover, lymphocytic infiltration was prominent after 4 weeks of GM treatment. However, the damage was seen more in the outer layer of the kidney cortex than in the medulla.

Renal tubules in the group treated with GM and Vit $\mathrm{E}+\mathrm{Se}$ combination showed accumulation of luminal debris by the end of the first week (Figure 2 d). In addition, lymphocyte infiltration after 4 weeks of co-administration of GM, Vit E and Se was almost similar to that seen in the kidney of rats exposed to GM only (Figure $2 \mathrm{e}$ ). At the end of the first week, calcification of the necrotic renal tubules was observed (Figure $2 \mathrm{f}$ ).

Relative to the ultrastructure of the control kidneys, renal cells of the proximal convoluted tubules (PCT) of rats that received GM only had ultrastructural alterations such as necrosis, cytoplasmic vacuolation and cellular debris (Figure $3 \mathrm{a}$ ). The changes extended to the distal convoluted tubules (DCTs) and collecting ducts which showed a lot of lysosomal structures and large phagocytic vacuoles (Figure $3 \mathrm{~b}$ ). The renal ultrastructure of rats in this group showed no significant recovery from the GM-induced alterations. 
Table 1: Blood biochemical analysis (mean \pm SD) at weeks 1 and 4

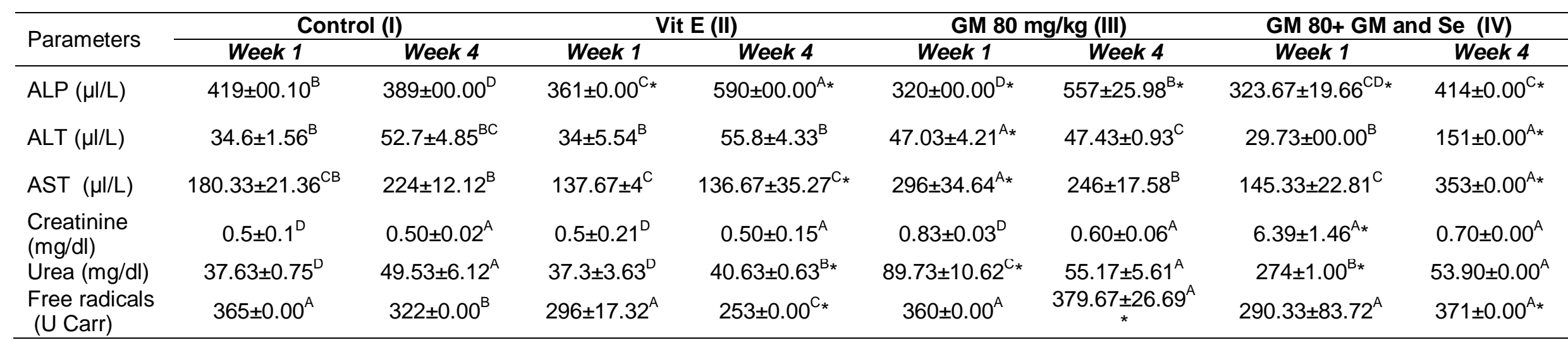

*Significant difference $(p<0.05$, compared with control group. Different letters within the same row indicate significant differences $(p<0.05)$ between experimental groups for the same biochemical parameter 

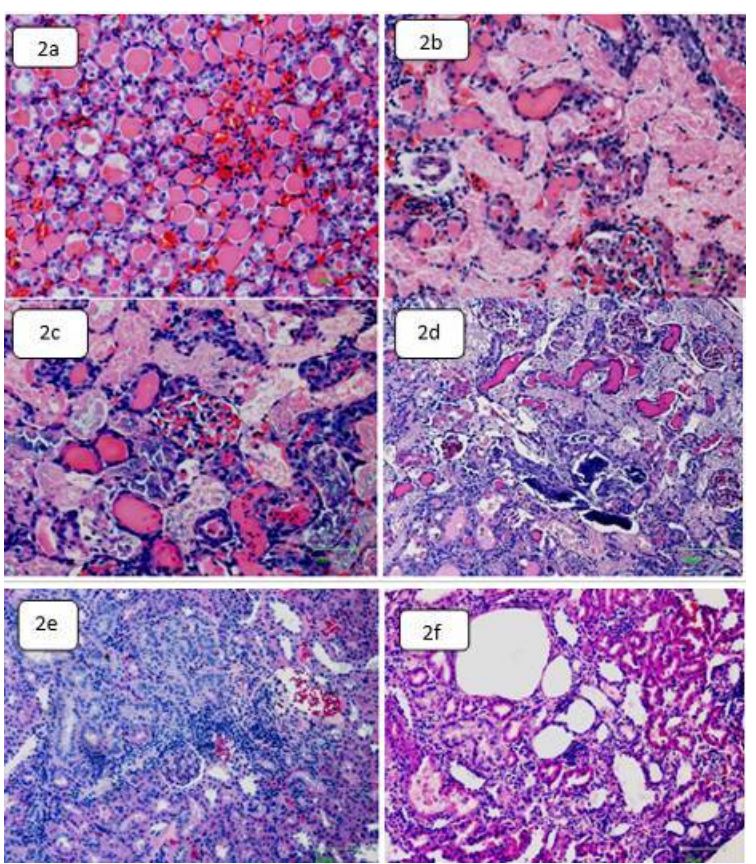

Figure 2: Photomicrographs demonstrating: (a) accumulation of eosinophilic materials in the collecting tubules of the medulla after one week of treatment with $\mathrm{GM}+$ Vit E + Se; (b) severe necrosis and accumulation of eosinophilic debris in the kidney convoluted tubules after one week of GM administration; (c) calcified necrotic tubules with intraluminal accumulation of necrotic debris after one week of treatment with GM + Vit E + Se); (d) accumulation of eosinophilic materials in the collecting tubules of the medulla after one week of GM+ Vit E + Se administration; (e) severe necrosis and accumulation of eosinophilic debris in the kidney convoluted tubules after one week of GM administration; (f) necrotic tubules with intraluminal accumulation of necrotic debris after one week of treatment with $\mathrm{GM}+\mathrm{Vit} \mathrm{E}+\mathrm{Se} .(\mathrm{H} \& \mathrm{E}, \times 400)$.

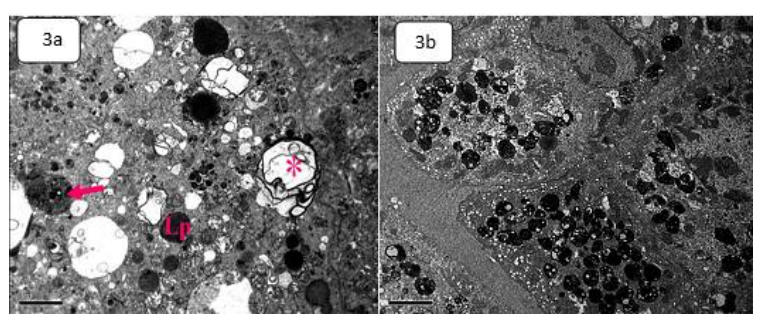

Figure 3: Electron micrograph showing epithelial cells lining the PCT of (a) GM-treated rat after one week, demonstrating necrosis and cytoplasmic vacuolations containing cellular debris; (b) Electron micrograph $\mathrm{GM}+$ Vit.E +Se-treated rats after one week, showing lysosomal structures and large phagocytic vacuoles $(\times 4000)$

\section{Hepatic histological alterations}

The hepatic tissues of the control rats demonstrated normal architecture with normal hepatic lobules, hepatocytes and hepatic portal triads (Figure 4a). In addition, the ultrastructure of the control liver appeared normal (Figure 4b). The hepatocytes showed normal nucleoplasm with nuclei surrounded by even distinct nuclear envelop showing heterochromatin adjacent to the border. The cytoplasm of these hepatocytes appeared crowded with mitochondria, endoplasmic reticulum and bounded ribosomes.

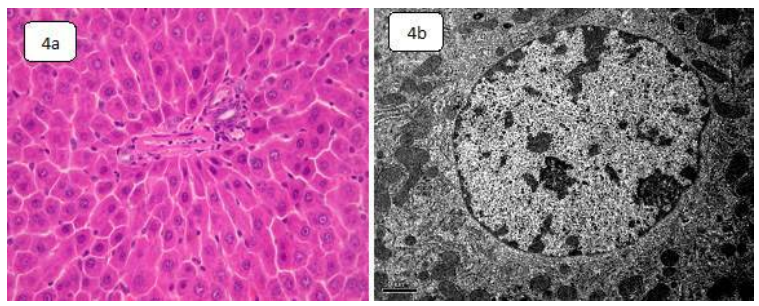

Figure 4: a- Photomicrographs showing liver of control rat with normal histological structure $(\mathrm{H} \& \mathrm{E} ; \times 400)$; bElectron micrograph showing liver of control rat with normal ultrastructure $(\times 6000)$

Rats treated with a combination of Vit $\mathrm{E}$ and $\mathrm{Se}$ had no significant changes in renal or hepatic tissues. In contrast, after 4 weeks of treatment, liver sections from GM-treated rats showed congestion and haemorrhagic foci accompanied by hepatic necrosis and lymphocytic infiltration (Figures $5 \mathrm{a}-5 \mathrm{c}$ ).
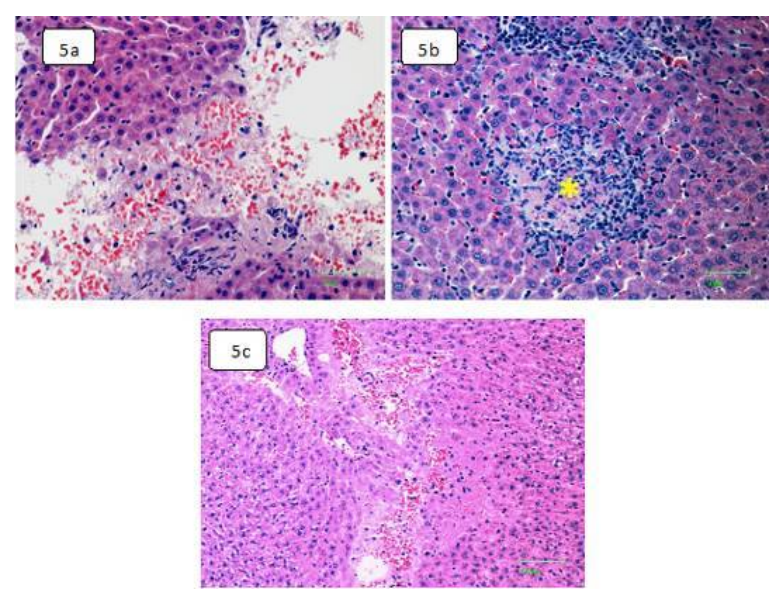

Figure 5: (a) Photomicrograph of liver section of GMtreated rats showing- scattered hemorrhagic-necrosis after one week of drug administration (H \& E; ×1000); (b) focal necrosis with lymphocytes infiltration microgranuloma) (*) after one week of GM administration $(\times 400)$; (c) haemorrhagic foci with necrosis after 4 weeks of treatment $(\mathrm{H} \& \mathrm{E} ; \times 400)$

Similar to rats exposed to GM only, rats in the group treated with GM in combination with Vit and Se had hepatocytes necrosis and lymphocytic infiltration (Figures 6a-d). 


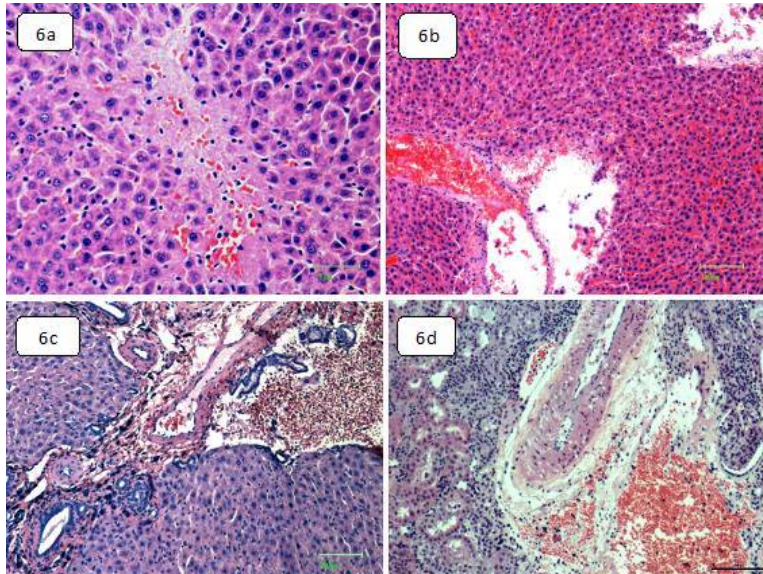

Figure 6: Photomicrograph of liver section from rats treated with $\mathrm{GM}+\mathrm{Vit} \mathrm{E}+\mathrm{Se}$ showing (a) necrosis at the end after one week (H \& E; x1000); (b) focal necrosis and pyknosis after one week (H \& $E ; x 1000)$; (c) hemorrhage with edema after 4 weeks ( $H$ \& E; $x 400)$; (d) hemorrhagic foci with necrosis after 4 weeks $(H$ \& E; $x 400)$.

\section{Ultrastructural changes}

Co-administration of GM and Vit E + Se led to mitochondrial swelling and crystolysis, necrosis, endoplasmic reticulum disruption and occasional cytoplasmic vacuolization, as shown on Figures $7 \mathrm{a}$ and $7 \mathrm{~b}$.

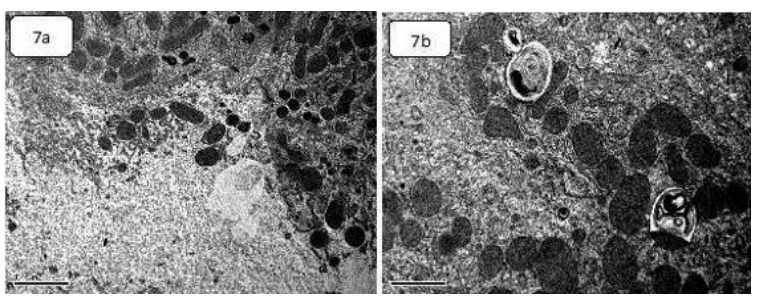

Figure 7: Electron micrographs of hepatocytes of rats treated with $\mathrm{Gm}+\mathrm{Vit} \mathrm{E}+\mathrm{Se}$ for 4 weeks showing (a) necrotic foci $(\times 5000)$; (b) swollen mitochondria with damaged cristae $(\times 10000)$

\section{DISCUSSION}

Gentamicin (GM)-induced toxicity limits chronic administration of GM. The present study investigated the in vivo chemoprotective role of oral Vit $\mathrm{E}$ and $\mathrm{Se}$ against GM-induced hepatotoxicity and nephrotoxicity. The findings revealed that daily administration of GM at a dose of $80 \mathrm{mg} / \mathrm{kg}$ for 4 weeks led to significant hepatotoxicity and nephrotoxicity in rats. In addition, the study showed that co-administration of oral Vit $\mathrm{E}$ and Se combination did not ameliorate the GM-induced toxicity. These findings may indicate that oral administration of Vit $E$ and $S e$ does not protect against GMinduced hepatotoxicity and nephrotoxicity.
Several studies have reported that GM caused hepatotoxic and nephrotoxic effects in experimental animals $[6,18]$. The results of the present study are in line with these previous reports, since GM induced significant toxicity after 1 week treatment, as shown in significant increases in serum levels of ALT, AST, creatinine and urea, and histological alterations in the hepatic and renal tissues of the rats. Interestingly, there were no significant differences in results of kidney and liver function tests between 1-week GM administration and 4week GM administration. This finding may indicate that 1-week GM administration is enough to induce significant hepatotoxic and nephrotoxic effects in rats. The observed renal histological changes, and the accompanying increased levels of ALP indicate functional disorders in the liver and kidney, as postulated by Kim and Moon study [19]. It has been reported that GM induced renal toxicity and significant elevation in ALP [3]. The hepatic alterations observed in histological examination in the present study confirm that significant structural changes in the liver lead to significant increase in the blood level of ALT.

Creatinine and urea are considered biomarkers of the efficiency of renal function [20]. Increased blood creatinine and urea are strongly related to renal damage [7]. Consistent with this relationship, it was found exposure to GM caused significant renal alterations and significant elevations in serum creatinine and urea. These results are in agreement with those obtained in an earlier study in which creatinine and urea levels were elevated after 5-7 days of GM administration [7].

The GM-induced hepatotoxicity and nephrontoxicity might be a result of the generation of reactive oxygen species which increase lipid peroxidation and lower the activities of antioxidant enzymes [21]. In addition, GM acts as an iron chelator by forming an iron-GM complex which is considered a potent catalyst of free radical generation [22]. Some studies have shown that antioxidant agents such as curcumin, Arabic gum, probucol, vitamin $\mathrm{C}$, lycopene and grape seed extract can be used to ameliorate GM-induced nephrotoxicity in rats $[18,21,22]$.

Vitamin $\mathrm{E}$ and Se are popularly used as antioxidants, and some studies have reported that these agents ameliorated drug-induced toxicity [14]. The present study found that combined treatment with Se and Vit. E lowered free radical levels, when compared to corresponding values for the control and GMtreated groups. However, co-administration of 
GM with Vit $\mathrm{E}$ and Se did not ameliorate the levels of free radicals, consistent with elevated levels of kidney and liver function biomarkers, and with abnormal histological alterations. These findings may indicate that the antioxidant effects of orally administrated Vit $\mathrm{E}$ and $\mathrm{Se}$ are not sufficient to protect the liver and kidney from oxidative stress induced by GM exposure. This may be due to the incomplete oral absorption of Vit $\mathrm{E}$ and $\mathrm{Se}$, or due to other reasons which need further investigation. The results of the current study agree with a previous report which showed that Vit E and diphenyl-phenylenediamine failed to offer protection against GM-induced nephrotoxicity [15]. However, the findings of the present study are in agreement with those of a previous study which indicated that Vit E attenuated GM-induced nephrotoxicity in rats [23]. It has been shown that Vit $\mathrm{E}$ and $\mathrm{Se}$ combination might not be beneficial to health and recovery from diseases. Moreover, it has been demonstrated that the use of Vit $\mathrm{E}$ and $\mathrm{Se}$ supplements did not offer any appreciable benefits in prevention of prostate cancer [24]. Indeed, Vit E and Se supplementation has been shown to significantly increase the risk of prostate cancer among healthy men [16]. The elevation of serum ALT and ALP levels as seen in the present study may indicate that Vit $E$ and Se may have harmful impact on the liver.

\section{CONCLUSION}

The results obtained in the present investigation indicate that exposure to GM is capable of inducing significant biochemical alterations, together with marked deleterious ultrastructural alterations in renal and hepatic tissues. However, oral administration of Vit $E$ and Se combination does not ameliorate GM-induced toxicity.

\section{DECLARATIONS}

\section{Acknowledgement}

The authors are thankful to the Deanship of Scientific Research and the Research Centre at College of Science of King Saud University; for supporting this research work.

\section{Conflict of interest}

No conflict of interest is associated with this work.

\section{Contribution of authors}

We declare that this work was done by the authors named in this article, and all liabilities pertaining to claims relating to the content of this article will be borne by the authors. Amin AlDoaiss did most of the laboratory work and wrote the manuscript. Yazun Jarrar did the statistical analysis, contributed to the interpretation of the results, and writing and reviewing the manuscript.

\section{Open Access}

This is an Open Access article that uses a funding model which does not charge readers or their institutions for access and distributed under the terms of the Creative Commons Attribution License (http://creativecommons.org/licenses/by/ 4.0) and the Budapest Open Access Initiative (http://www.budapestopenaccessinitiative.org/rea d), which permit unrestricted use, distribution, and reproduction in any medium, provided the original work is properly credited.

\section{REFERENCES}

1. Nagai J, Takano M. Molecular aspects of renal handling of aminoglycosides and strategies for preventing the nephrotoxicity. Drug Metab Pharmacokinet 2004; 19(3): 159-170.

2. Takahashi S, Xu C, Sakai T, Fujii K, Nakamura M. Infective endocarditis following urinary tract infection caused by Globicatella sanguinis. IDCases 2018; 11: 18-21.

3. Alarifi S, Al-Doaiss A, Alkahtani S, Al-Farraj SA, Al-Eissa MS, Al-Dahmash B, Al-Yahya H, Mubarak M. Blood chemical changes and renal histological alterations induced by gentamicin in rats. Saudi J Biol Sci 2012; 19(1): 103-110.

4. Pedraza-Chaverri J, Maldonado PD, Medina-Campos ON, Olivares-Corichi IM, Granados-Silvestre MA, Hernandez-Pando $R$, Ibarra-Rubio ME. Garlic ameliorates gentamicin nephrotoxicity: relation to antioxidant enzymes. Free Radic Biol Med 2000; 29(7): 602-611.

5. Cuzzocrea S, Mazzon E, Dugo L, Serraino I, Di Paola R, Britti D, De Sarro A, Pierpaoli S, Caputi A, Masini E, et al. A role for superoxide in gentamicin-mediated nephropathy in rats. Eur J Pharmacol 2002; 450(1): 6776.

6. Manikandan R, Beulaja M, Thiagarajan R, Priyadarsini A, Saravanan $R$, Arumugam M. Ameliorative effects of curcumin against renal injuries mediated by inducible nitric oxide synthase and nuclear factor kappa $B$ during gentamicin-induced toxicity in Wistar rats. Eur $J$ Pharmacol 2011; 670(2-3): 578-585.

7. Karahan I, Atessahin A, Yilmaz S, Ceribasi AO, Sakin F. Protective effect of lycopene on gentamicin-induced oxidative stress and nephrotoxicity in rats. Toxicology 2005; 215(3): 198-204. 
8. Nabavi SF, Nabavi SM, Moghaddam AH, Naqinezhad A, Bigdellou R, Mohammadzadeh S. Protective effects of Allium paradoxum against gentamicin-induced nephrotoxicity in mice. Food Funct 2012; 3(1): 28-29.

9. Lobo V, Patil A, Phatak A, Chandra N. Free radicals, antioxidants and functional foods: Impact on human health. Pharmacogn Rev 2010; 4(8): 118-126.

10. Kurutas EB. The importance of antioxidants which play the role in cellular response against oxidative/nitrosative stress: current state. Nutr J 2016; 15(1): 71.

11. Gonca S, Ceylan S, Yardimoglu M, Dalcik H, Yumbul Z, Kokturk S, Filiz S. Protective effects of vitamin $E$ and selenium on the renal morphology in rats fed highcholesterol diets. Pathobiology 2000; 68(6): 258-263.

12. Monteiro DA, Rantin FT, Kalinin AL. The effects of selenium on oxidative stress biomarkers in the freshwater characid fish matrinxa, Brycon cephalus (Gunther, 1869) exposed to organophosphate insecticide Folisuper 600 BR (methyl parathion). Comp Biochem Physiol C Toxicol Pharmacol 2009; 149(1): 4049.

13. Karabulut-Bulan O, Bolkent $S$, Yanardag R, BilginSokmen $B$. The role of vitamin $C$, vitamin $E$, and selenium on cadmium-induced renal toxicity of rats. Drug Chem Toxicol 2008; 31(4): 413-426.

14. Aboul-Soud MA, Al-Othman AM, El-Desoky GE, AlOthman ZA, Yusuf K, Ahmad J, Al-Khedhairy AA. Hepatoprotective effects of vitamin E/selenium against malathion-induced injuries on the antioxidant status and apoptosis-related gene expression in rats. J Toxicol Sci 2011; 36(3): 285-296.

15. Ramsammy LS, Josepovitz C, Ling $K Y$, Lane BP, Kaloyanides GJ. Failure of inhibition of lipid peroxidation by vitamin $E$ to protect against gentamicin nephrotoxicity in the rat. Biochem Pharmacol 1987; 36(13): 2125-2132.
16. Klein EA, Thompson IM, Jr., Tangen CM, Crowley JJ, Lucia MS, Goodman PJ, Minasian LM, Ford LG, Parnes $H L$, Gaziano JM, et al. Vitamin $E$ and the risk of prostate cancer: the Selenium and Vitamin E Cancer Prevention Trial (SELECT). JAMA 2011; 306(14): 1549-1556.

17. Rowsell HC. The Canadian Council on Animal Care--its guidelines and policy directives: the veterinarian's responsibility. Can J Vet Res 1991; 55(3): 205.

18. Tavafi M, Ahmadvand $H$. Effect of rosmarinic acid on inhibition of gentamicin induced nephrotoxicity in rats. Tissue Cell 2011; 43(6): 392-397.

19. Kim SY, Moon A. Drug-induced nephrotoxicity and its biomarkers. Biomol Ther (Seoul) 2012; 20(3): 268-272.

20. Abdel-Raheem IT, El-Sherbiny GA, Taye A. Green tea ameliorates renal oxidative damage induced by gentamicin in rats. Pak J Pharm Sci 2010; 23(1): 21-28.

21. Yaman I, Balikci E. Protective effects of nigella sativa against gentamicin-induced nephrotoxicity in rats. Exp Toxicol Pathol 2010; 62(2): 183-190.

22. Khan SA, Priyamvada S, Farooq N, Khan S, Khan MW, Yusufi AN. Protective effect of green tea extract on gentamicin-induced nephrotoxicity and oxidative damage in rat kidney. Pharmacol Res 2009; 59(4): 254262.

23. Kadkhodaee M, Khastar H, Faghihi M, Ghaznavi R, Zahmatkesh $M$. Effects of co-supplementation of vitamins $E$ and $C$ on gentamicin-induced nephrotoxicity in rat. Exp Physiol 2005; 90(4): 571-576.

24. Lippman SM, Klein EA, Goodman PJ, Lucia MS, Thompson IM, Ford LG, Parnes HL, Minasian LM, Gaziano JM, Hartline JA, et al. Effect of selenium and vitamin $E$ on risk of prostate cancer and other cancers: the Selenium and Vitamin E Cancer Prevention Trial (SELECT). JAMA 2009; 301(1): 39-51. 